\title{
Diagnostic and therapeutic values of percutaneous trans-hepatic cholangiography (PTC) and biliary drainage in obstructive jaundice patients
}

\author{
Dr. Waleed Said Abo Shanab, Lecturer of diagnostic radiology, faculty of medicine, \\ Port Said University \\ Dr. Carmen Ali Zarad, (corresponding author) Lecturer of diagnostic radiology, faculty \\ of medicine, Port Said University
}

\begin{abstract}
$\underline{\text { Abstract }}$
Background: Percutaneous trans-hepatic cholangiography is an interventional diagnostic and therapeutic procedure used in the diagnosis and management of obstructive jaundice. The aim of the study was to evaluate the diagnostic and therapeutic values of percutaneous trans-hepatic cholangiography and biliary drainage in obstructive jaundice patients.

Patients and Methods: Study included 30 patients with obstructive jaundice. All patients performed percutaneous trans-hepatic cholangiography. Diagnostic percutaneous transhepatic cholangiography was done first to detect the site, level and shape of the stenosis then therapeutic Percutaneous biliary drainage was done to drain the bile from the dilated biliary tree.

Results: This study included 30 patients with obstructive jaundice (21 male and 9 females). $96.66 \%$ of patients had malignant obstruction and $3.33 \%$ had benign obstruction. The accuracy of PTC in diagnosis of biliary dilatation, cause and level of
\end{abstract}


obstruction was $100 \%$. External biliary drainage done for all 30 patients then internal stent was inserted for 26 patients with technical success rate for passing the stricture was $86.66 \%$. Technical success rate of percutaneous biliary drainage was $100 \%$ manifested by decrease in the serum bilirubin level and improvement of liver function tests in all patients 1 week after the drainage. Technique-related morbidity was $13.33 \%$ with no procedure-related mortality

Conclusion: Percutaneous trans-hepatic cholangiography has high diagnostic accuracy rate $100 \%$ in diagnosis of biliary dilatation, cause and level of biliary obstruction. Percutaneous trans-hepatic biliary drainage has high therapeutic success rate of bile drainage $100 \%$ with high technical success rate $86.66 \%$ for passing the stricture in patients with obstructive jaundice.

Key words: Percutaneous trans-hepatic biliary drainage; percutaneous trans-hepatic cholangiography; obstructive jaundice.

\section{Introduction}

Obstructive jaundice has many etiologies; it may be caused by benign or malignant causes. Obstruction of biliary system leads to impairment of the patient's quality of life and cause cholangitis, pruritus, and may ends by liver cell failure (Chandrashekhara et.al. 2016).

The most common causes of malignant obstructive jaundice are cholangiocarcinoma, gall bladder carcinoma, pancreatic carcinoma, metastatic neoplasms and malignant lymph node enlargement that cause compression of the common bile duct (CBD) (Rees et.al. 2020).

Obstructive jaundice diagnosis is made by many invasive and noninvasive methods: noninvasive methods involved ultrasonography, CT and magnetic resonance cholangiopancreatography (MRCP) and invasive methods involved percutaneous transhepatic cholangiography (PTC) and endoscopic retrograde cholangio-pancreatography (ERCP), these two techniques have diagnostic and therapeutic values (Dawoud et.al. 2019).

The most of lesions of malignant obstructive jaundice are unresectable and advanced. In these cases, palliative treatment is the only choice to drain the obstruction, relief the pain and improved the patient quality of life (Ray et.al. 2013). Palliative biliary drainage can be used also as a tool for palliative intra-biliary chemotherapy injection and improving quality of life in advanced pancreatic and biliary tract cancers (Rees et.al. 2020).

Drainage of bile in obstructive jaundice can be done surgically by ERCP or by PTC. The methods used for drainage is detected according to the site of cancer and whether it is 
operable or not. The use of PTC accelerates internal and external biliary drainage and it is considers as the main technique of relieving biliary tract obstruction for malignant obstructive jaundice that occurs above the level of the common hepatic duct, also PTC can be used when ERCP unable to relieve distal malignant biliary tract obstruction (Crosara Teixeira et.al. 2013).

PTC is an invasive technique requiring transhepatic insertion of a needle into a dilated bile duct, followed by injection of contrast material to opacify the bile ducts. PTC is usually performed for evaluation of patients who are found to have biliary duct dilatation on ultrasonography or other imaging test and who are not candidates for endoscopic retrograde cholangiopancreatography (ERCP) (Ahmed et al., 2016).

PTC plays an important role in the diagnosis and treatment of biliary disease. Recently PTC is used in diagnosis and treatment of biliary obstruction in many cases when endoscopic methods are failed or are not suitable (Shawyer et al., 2013).

PTC allows a number of therapeutic interventions for benign causes of obstructive jaundice, as it used in drainage of infected bile in case of cholangitis, dilation of benign biliary strictures or extraction of biliary tract stones (Altman \& Zangan., 2016).

PTC is an intervention technique that has high incidence of morbidity and mortality, with many major complications, these complications include blockage of the stent dislodgement of the drain and the need to repeat the technique. Other possible complications include cholangitis, pain, biliary leakage, hemorrhage, and sepsis (Tuqan et.al.2017).

\section{Patients and Methods}

\section{Patients:}

This study performed in diagnostic radiology department of our institution. This study included 30 patients ( 21 males and 9 females with average age ranging from 44 to 70 years with an average of $59.1 \pm 6.3$ years) during the period between May 2019 to May 2020 to investigate the therapeutic and diagnostic value of percutaneous transhepatic cholangiography and biliary drainage in patients with obstructive jaundice.

Diagnosis of obstructive jaundice was done by US, CT and MRI in some cases.

The inclusion criterion were patients with obstructive jaundice caused by non-operable biliary diseases, and they could not be treated endoscopically. Written consent was taken from all patients that gave us permission to make the procedures.

Exclusion criteria were presence of significant ascites, history of previous biliary stent insertion, history of previous biliary surgery or radiotherapy, history of allergy to any 
medications, especially antibiotics or iodine, an (international normalized ratio (INR) more than1.5), and a platelet count less than $70,000 \times 10^{3}$.

\section{Methods:}

All 30 patients included in the study performed percutaneous transhepatic cholangiography.

Before the procedure, all patients were subjected to the history taking, clinical examination radiological and laboratory investigation.

Laboratory investigations included complete blood picture, blood glucose, cholesterol, triglyceride and uric acid levels, prothrombin time, partial thromboplastin time, liver and renal function tests.

Radiological investigations before procedure included US, CT and if needed MRCP. An intravenous line was done for all patients.

Diagnostic PTC was done first to detect the site, level and shape of the stenosis then therapeutic Percutaneous biliary drainage was done in all patients to drain the bile from the dilated biliary tree.

Two approaches were used for PTC, the right hepatic lobe approach, and the left hepatic lobe approach. Left hepatic lobe approach was chosen in 6 cases (two cases, the left approach was used alone due to the presence of ascites, and in 4 cases, the left approach was used in combination with the right hepatic approach) while the right hepatic approach alone was used in the remaining 24 cases. The 8 Fr percutaneous drainage catheters were remained after stenting in all cases for 3 days.

PTC were done for all patients by using 21 or $22 \mathrm{G}$ Chiba needle under local anesthesia with mild intravenously sedation and analgesia.

Patients lie in supine position and in right hepatic lobe approach the puncture site was done under the right tenth rib in the mid axillary line with the patient in full inspiration. Under fluoroscopy, insertion of a $21 \mathrm{G}$ needle was done and advanced the needle with angulation 10 degree cranially and forward for a distance $3-4 \mathrm{~cm}$ under ultrasound guide the needle was directed to the suitable intrahepatic duct. Injection of contrast media during withdrawn of the needle was done till visualization of the bile duct. After entering the intrahepatic bile duct insertion of platinum tip guidewire and angiographic catheter through the needle into the biliary system were done. The needle then removed when the guidewire reached the duodenum.

Patients in which the biliary obstruction site was crossed an 8 Fr ring biliary catheter was inserted and for the initial days both external biliary drainage and internal biliary 
drainage into the duodenum were done. After achievement of successful drainage, internal drainage was left, and the catheter was capped externally. If we could not cross the site of obstruction, external drainage catheter was left for drainage of the biliary system.

The same steps were done for left hepatic lobe approach except for the puncture site was in subxiphoid position.

All procedures of PTC were performed using, AXIOM Artis MP, SIEMENS MEDICAL SYSTEMS, ERLANGEN, GERMANY, 2001. All US examinations were done with General electric medical system apparatus (LOGIQ 9). All PTC were done by Philips SD 800 apparatus.

Percutaneous insertion of biliary stents was done in 26 patients, 21 of them were by metallic stents (Viabil covered metallic stents, diameter 8-10 mm, length 6,8 , or $10 \mathrm{~cm}$ ) and the remaining 5 patients were by plastic stents.

Percutaneous transhepatic external biliary drainage was done in 4 patients with no other procedure.

After the procedure, all patients were checked for any problems in the heart rate, body temperature, breathing and blood pressure. After the procedure many medications was given to the patient as analgesics for pain and antibiotics for a week to prevent sepsis.

Patients were classified according to the cause of obstruction into patients with benign lesions and patients with malignant lesions.

Patients were classified according to the obstruction level into proximal or distal obstruction. Proximal obstruction was further classified into three types (Type I, II and III) according to bisthmus-correlate classification.

\section{$\underline{\text { Statistical Methods }}$}

All data are collected, represented, summarized, and analyzed by using appropriate statistical tests using SPSS, version statistical program.

For qualitative data, all data were represented by number and percentage and were analyzed by using chi-square statistical test.

For qualitative data, all data were summarized by mean standard deviation and were analyzed by using independent $\mathrm{t}$-test.

Multivariate analysis logistic regression modules were used. 


\section{$\underline{\text { Results }}$}

This study included 30 patients (21 male and 9 females; mean age, $59.1 \pm 6.3$ years; range, 44 - 70 years) with obstructive jaundice.

Of these 30 patients, 29 patients (96.66\%) had malignant pathology \{15 patients (50\%) with cholangiocarcinoma, 5 patients $(16.66 \%)$ with hepatocellular carcinoma, 5 patients (16.66\%) with gastric or recurrent gastric carcinoma (figure 1), 3 patients (10\%) with colonic carcinoma (figure 2) and liver metastasis and 1 patient (3.33\%) pancreatic carcinoma (figure 3 ) $\}$ and one patient $(3.33 \%$ ) had benign pathology for obstruction and is due to post-cholecystectomy choledochal stricture (figure 4).

The diagnoses were based on laboratory diagnosis and imaging studies including US, CT, MRCP in some cases and finally Percutaneous Transhepatic Cholangiography. The accuracy of US and CT in diagnosis of biliary dilatation were $100 \%$ for both techniques, their accuracy for diagnosis of cause of obstruction were $56.6 \%$ and $80 \%$ respectively, and their accuracy for diagnosis of level of obstruction were $46.6 \%$ and $66.6 \%$ respectively. The accuracy of PTC in diagnosis of biliary dilatation, cause and level of obstruction was $100 \%$ (table 1).

According to the level of obstruction the patients were classified according to bisthmuscorrelate classification there were 22 patients $(73.33 \%)$ with type I obstruction, one patient $(3.33 \%)$ with type II obstruction, one patient (3.33\%) with type III obstruction and 6 patients $(20 \%)$ had distal obstruction.

Successful relieve of obstruction were occur in 30 patients (100\%). In these 30 patients, relieve of obstruction was achieved by means of percutaneous external biliary drainage and/or biliary stenting. Temporary external biliary drainage done for all 30 patients, and it followed by internal stent drainage in 26 patients $(86.66 \%$ ) (metallic stent in 21 patients and plastic stent in 5 patients) with technical success rate for passing the stricture was $86.66 \%$. From these 26 patients 25 patients $(83.33 \%$ ) had successful biliary stenting in one setting and in one patient $(3.33 \%)$ an external percutaneous biliary drainage (PTBD) with 6-Fr pigtail catheter was established then the lesion was stented 7 days later. Failure of internal biliary stenting occurred in 4 patient (13.33\%) and draining the biliary system were done by simple external catheter only (table 2 ).

Right hepatic lobe approach was done in 24 patients (80\%), left hepatic lobe approach done in 2 patients $(6.66 \%)$ and both approaches done in 4 patients (13.33\%) (table 2).

Single stent placement was adequate to pass the stricture in 25 patients $(83.33 \%)$ (20 patients with type I stricture according to Bismuth- Corlette classification and 5 patients with distal obstruction). Bilateral stenting with 2 stents was employed in 1 patient (3.33\%) with type IIIa stricture. The technical failure in the four cases $(13.33 \%)$ was due 
to hilar cholangiocarcinoma in two patients $(6.66 \%)$ (Bismuth type I), hepatoma in one patient $(3.33 \%)$ (Bismuth type II) and advanced gastric carcinoma in the remained one (3.33) (Bismuth type I), and this four patients show non-identifiable passage to the extrahepatic biliary tract (table 2 ).

Technique-related complications occurred in 4 of the 30 cases (13.33\%). There was one case of arterial injury with subsequent hemorrhage that occurred during PTC needle insertion. Angiography was performed and revealed contrast extravasation from the right hepatic artery branch, which was immediately embolized with metallic coils and successful stenting followed 7 days later, once the patient's general condition had improved. In another case bile leakage and subsequent bile peritonitis, but this controlled by adequate medical treatment. In the 3rd case developed a self-limiting sub capsular liver hematoma. In one late complication occurred after 7 days and was due to acute cholangitis and the condition was controlled by medical treatment, (table 3). Thus, technique-related morbidity was $13.33 \%$, with a procedure-related mortality rate of $0 \%$.

Occlusion occur in 8/26 patients (30.07\%) (6 cases due to tumor growth and 2 case due to sludge incrustation which relieved with a semi-inflated balloon). In the 6 cases of tumor growth re-stenting by metallic stent were occur in 4 cases $(15.38 \%)$, while in the remaining two cases $(7.69 \%)$, who were metallic, this two stents were remained inside and drainage was done in one case $(3.84 \%)$ by simple pigtail catheter draining the bile internally and externally, while in the other case $(3.84 \%)$, the draining were occur externally by simple catheter.

Various symptoms caused by jaundice, such as anorexia, itching, nausea, abdominal pain, and fever, were relieved in all the patients within one week after PTBD. Resolution of jaundice was confirmed biochemically by a decline in bilirubin one week after intervention.

The mean total bilirubin level was $21.8 \pm 3.46 \mathrm{mg} / \mathrm{dL}$, (ranging from 13.5 to $27.5 \mathrm{mg} / \mathrm{dL}$ ) prior to the biliary drainage and became $4.19 \pm 1.84 \mathrm{mg} / \mathrm{dL}$ (ranging from 1.2 to 7.8 $\mathrm{mg} / \mathrm{dL}$ ) one week after the biliary drainage procedure ( $\mathrm{p}$-value 0.001 ). The mean direct bilirubin level was $17.8 \pm 3.4 \mathrm{mg} / \mathrm{dL}$, (ranging from 10.4 to $25.4 \mathrm{mg} / \mathrm{dL}$ ) prior to the biliary drainage and became $2.62 \pm 1.15 \mathrm{mg} / \mathrm{dL}$, (ranging from 0.5 to $4.8 \mathrm{mg} / \mathrm{dL}$ ) one week after the biliary drainage procedure (p-value 0.0003 ). this decrease in bilirubin levels was statistically significant.

Technical success rate of biliary drainage was 30/30 (100\%), adequate biliary drainage was achieved in 30 patients, decrease in the serum bilirubin level, alkaline phosphatase and improvement of liver function tests was seen in all (30 out of 30) of our patients following 1 week after percutaneous biliary drainage. 
Table (1) : Validity of different diagnostic tools in diagnosis of obstructive jaundice.

\begin{tabular}{|c|c|c|c|c|}
\hline & $\mathbf{U S}$ & CT & PTC & Others \\
\hline Cholangiocarcinoma $(n=15)$.) & & & & Histopathology is \\
\hline Biliary dilatation & $15 / 15(100 \%)$ & $15 / 15(100 \%)$ & $15 / 15(100 \%$ & needed in diagnosis o \\
\hline Cause & $8 / 15(53.3 \%)$ & $11 / 15(73.3 \%)$ & $15 / 15(100 \%)$ & $(7 / 15)$ of the \\
\hline Level of obstruction & $8 / 15(53.3 \%)$ & $11 / 15(73.3 \%)$ & $15 / 15(100 \%)$ & $\begin{array}{l}\text { cases and MRCP in } \\
(6 / 15) \text { of the cases. }\end{array}$ \\
\hline Hepatoma $(n=5)$ & & & & Histopathology is \\
\hline Biliary dilatation & $5 / 5(100 \%)$ & $5 / 5(100 \%)$ & $5 / 5(100 \%)$ & needed in diagnosis $o$ \\
\hline Cause & $3 / 5(60 \%)$ & $4 / 5(80 \%)$ & $5 / 5(100 \%)$ & $(1 / 5)$ cases and \\
\hline Level of obstruction & $3 / 5(60 \%)$ & $3 / 5(60 \%)$ & $5 / 5(100 \%)$ & $\begin{array}{l}\text { MRCP in }(2 / 5) \text { of the } \\
\text { cases. }\end{array}$ \\
\hline $\begin{array}{l}\text { Gastric or recurrent gastric } \\
\operatorname{mass}(n=5)\end{array}$ & & & & $\begin{array}{c}\text { Histopathology is } \\
\text { needed in diagnosis }\end{array}$ \\
\hline Biliary dilatation & $5 / 5(100 \%)$ & $5 / 5(100 \%)$ & $5 / 5(100 \%)$ & of $(2 / 5)$ of the cases. \\
\hline Cause & $3 / 5(60 \%)$ & $4 / 5(80 \%)$ & $5 / 5(100 \%)$ & \\
\hline Level of obstruction & $2 / 5(40 \%)$ & $3 / 5(60 \%)$ & $5 / 5(100 \%)$ & \\
\hline $\begin{array}{l}\text { Colonic carcinoma with liver } \\
\text { metastasis }(\mathbf{n}=\mathbf{3}) \text {. } \\
\text { Biliary dilatation } \\
\text { Cause } \\
\text { Level of obstruction }\end{array}$ & $\begin{array}{l}3 / 3(100 \%) \\
2 / 3(66.6 \%) \\
1 / 3(33.3 \%)\end{array}$ & $\begin{array}{l}3 / 3(100 \%) \\
3 / 3(100 \%) \\
2 / 3(66.6 \%)\end{array}$ & $\begin{array}{l}3 / 3(100 \%) \\
3 / 3(100 \%) \\
3 / 3(100 \%)\end{array}$ & $\begin{array}{l}\text { Histopathology is } \\
\text { needed in diagnosis o } \\
(2 / 3) \text { of the cases and } \\
\text { MRCP in }(2 / 3) \text { of the } \\
\text { cases. }\end{array}$ \\
\hline $\begin{array}{l}\text { Pancreatic carcinoma }(\mathbf{n}=\mathbf{1}) \text {. } \\
\text { Biliary dilatation } \\
\text { Cause } \\
\text { Level of obstruction }\end{array}$ & $\begin{array}{c}1 / 1(100 \%) \\
1 / 1(100 \%) \\
\text { Failed }\end{array}$ & $\begin{array}{c}1 / 1(100 \%) \\
1 / 1(100 \%) \\
\text { Failed }\end{array}$ & $\begin{array}{l}1 / 1(100 \%) \\
1 / 1(100 \%) \\
1 / 1(100 \%)\end{array}$ & $\begin{array}{l}\text { MRCP was done } \\
\text { and diagnose the } \\
\text { cause and level } \\
\text { of obstruction. }\end{array}$ \\
\hline $\begin{array}{l}\text { Post cholecystectomy biliary } \\
\text { stricture }(\mathbf{n}=\mathbf{1}) \text {. } \\
\text { Biliary dilatation } \\
\text { Cause } \\
\text { Level of obstruction }\end{array}$ & $\begin{array}{c}1 / 1(100 \%) \\
\text { Failed } \\
\text { Failed }\end{array}$ & $\begin{array}{c}\text { 1/1 }(100 \%) \\
\text { Failed } \\
\text { Failed }\end{array}$ & $\begin{array}{l}1 / 1(100 \%) \\
1 / 1(100 \%) \\
1 / 1(100 \%)\end{array}$ & $\begin{array}{l}\text { MRCP was done } \\
\text { and diagnose the } \\
\text { cause and level } \\
\text { of obstruction. }\end{array}$ \\
\hline
\end{tabular}


Table (2): Distribution of patients according to approach, success of the procedures (stent placement) and number of stents.

\begin{tabular}{|c|c|c|}
\hline & Number & $\%$ \\
\hline Approach. & $24 / 30$ & $80 \%$ \\
Right & $2 / 30$ & $6.66 \%$ \\
Left & $4 / 30$ & 13.33 \\
Right and left & $26 / 30$ & $86.66 \%$ \\
\hline Success rate of stent placement. & & \\
\hline Number of stents & $1.03 \pm 0.55$ & \\
X \pm SD & $0-2$ & \\
Range & & $13.33 \%$ \\
Number of stents & $4 / 30$ & $83.3 \%$ \\
0 & $25 / 30$ & $3.33 \%$ \\
\hline 2 & $1 / 30$ & \\
\hline
\end{tabular}

$\mathrm{X} \pm \mathrm{SD}=$ Mean \pm Standared deviation

$\mathrm{ERCP}=$ Endoscopic Retrograde Cholangiopancreatography

Table (3):Distribution of patients according to complications.

\begin{tabular}{|l|c|c|}
\hline & Number & $\%$ \\
\hline Complications: & & \\
Related to the procedures & $4 / 30$ & $13.33 \%$ \\
Late complications(stent t occlusions & $8 / 26$ & $30.07 \%$ \\
- Due to malignant growth. & $6 / 26$ & $23.07 \%$ \\
- Others. & $2 / 26$ & $7.69 \%$ \\
\hline
\end{tabular}




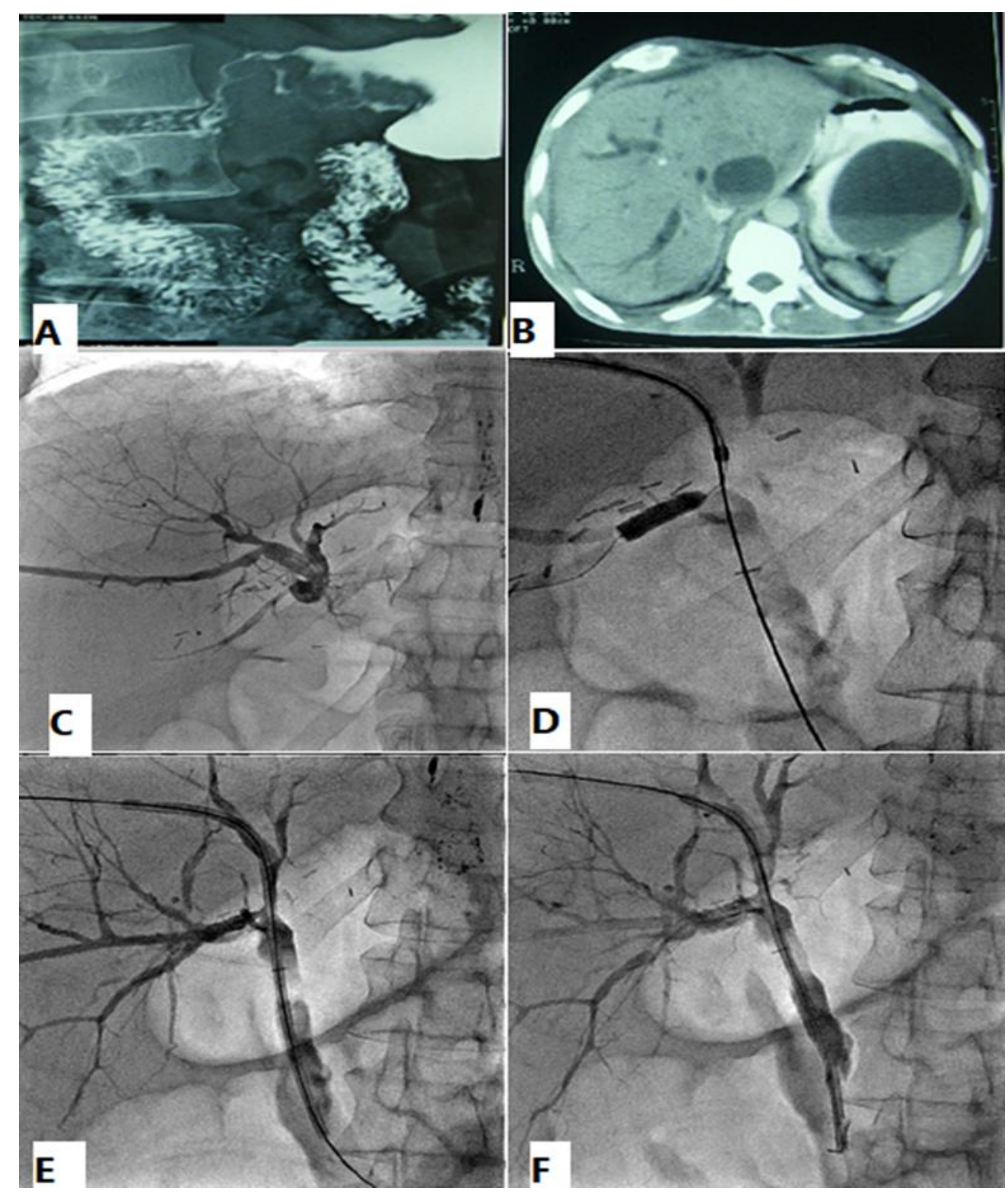

Figure (1): 55 years old male patient with obstructive jaundice due to porta-hepatis metastatic mass from gastric cancer. (A) Barium meal shows large irregular filling defect is seen in the pyloric region with shouldering and mucosal destruction (malignant neoplastic mass in the pyloric region). (B) ) Contrast enhanced axial CT scan shows hypodense mass seen at the porta hepatis with dilated intrahepatic biliary radicals, also there is large mass in the pyloric region of the stomach with marked wall thickening. (C) PTC shows obstruction at the porta hepatis below the bifurcation of the CBD (Bisthmus type I obstruction) (D) PTC shows guide wire is tried to pass the stricture (E) PTC shows the guide wire success to pass the stricture to the duodenum with the plastic stent over it. (F) PTC shows the guide wire is gradually withdrawn leaving the catheter passing the stricture to the duodenum. 

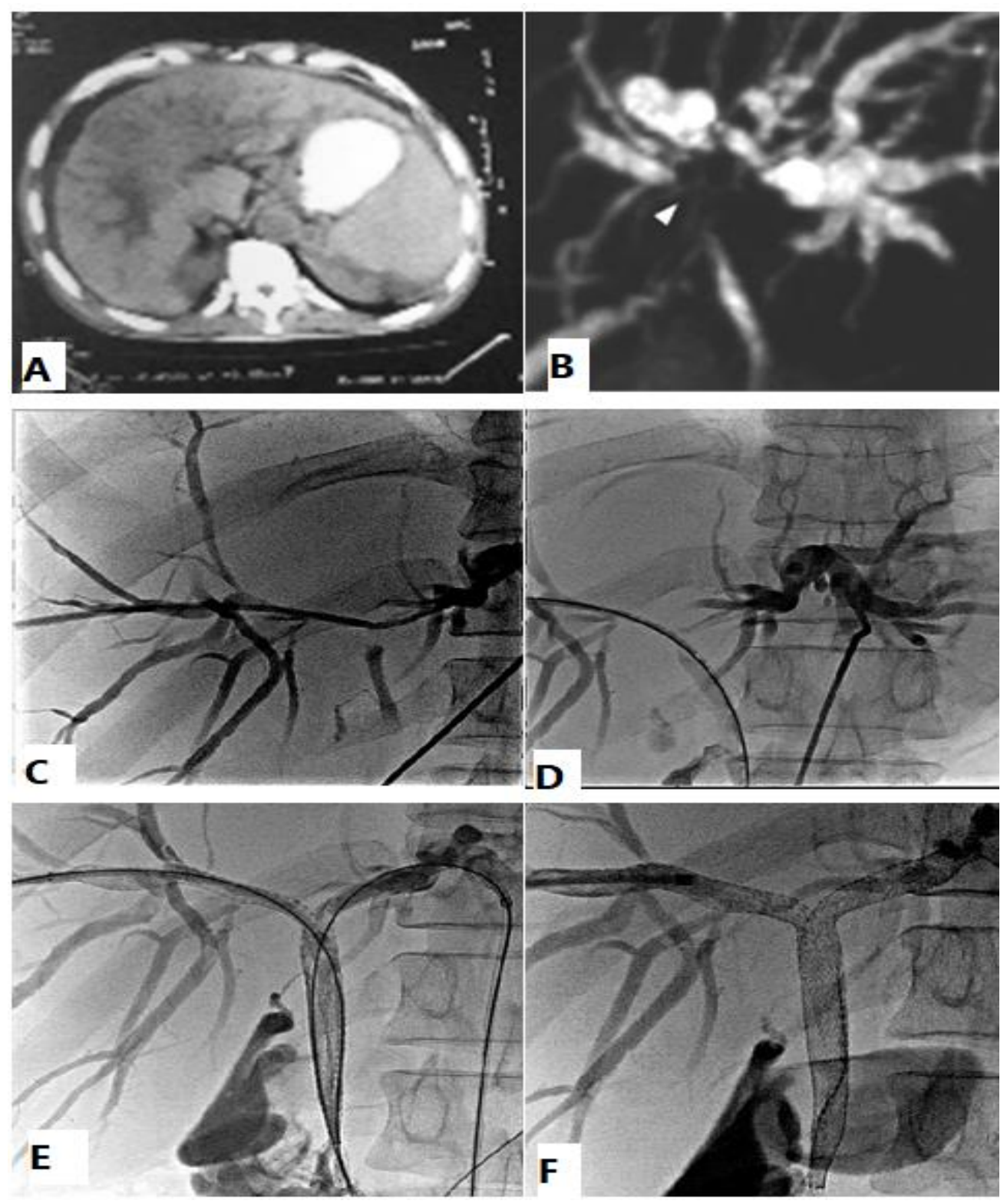

Figure (2): 64 years old male patient with obstructive jaundice due to porta-hepatis metastatic mass from cancer colon. (A). Enhanced axial CT scans shows an ill-defined low-density mass lesion at the porta hepatic (B). MRCP shows dilated biliary radicals, dilated right and left hepatic ducts with a mass at the site of union. (C\& D) PTC shows obstruction at the porta-hepatis with dilated and separate right and left systems of the biliary tree (Bisthmus type II stricture), with two guide wires placed from the dilated right and left systems (E) PTC shows the two guide wires are crossing the stricture to the duodenum with two metallic stents over it. (F) PTC shows removal of the two guide wires leaving the successfully placed metallic stents and contrast injection ensure that the stents were traversing the stricture to the duodenum. 

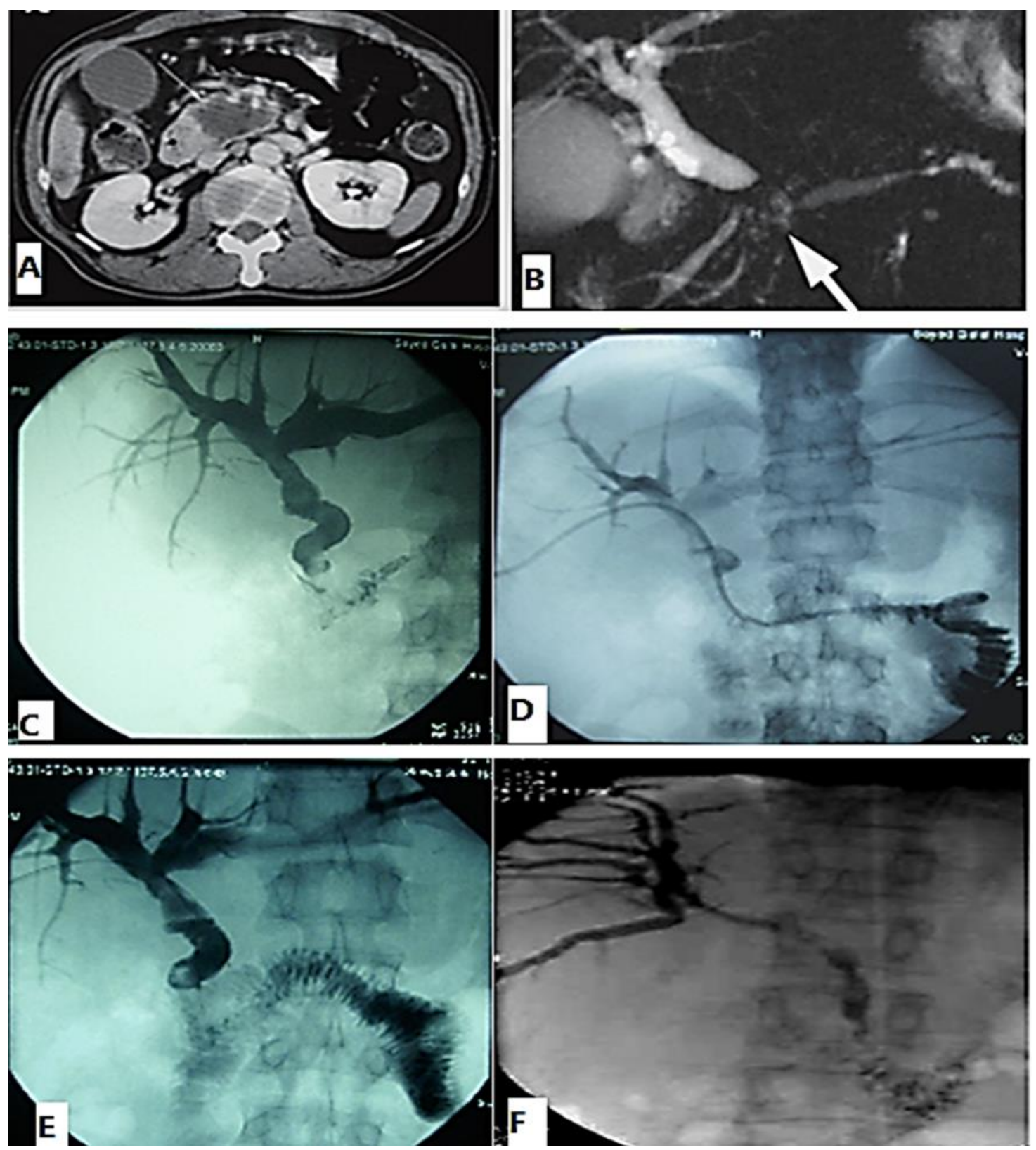

Figure (3): 65 years old male patient with pancreatic head carcinoma. (A). Enhanced axial CT scans show a low-density mass (arrow) at the head of pancreas (B). MRCP shows dilatation of the proximal main pancreatic and common bile duct with an abrupt obliteration of the ducts (arrow). (C). PTC shows obstruction at the distal part of the CBD. (D). PTC shows guide wire placed in the duodenum across the stricture with pigtail catheter over it. (E) PTC shows contrast injection ensure that the guide wire and the catheter are traversing the stricture to the duodenum, then the catheter is removed and the stent placement is taking place. (F). PTC following successful stent placement, flow of contrast through the obstructed segment to the duodenum. 


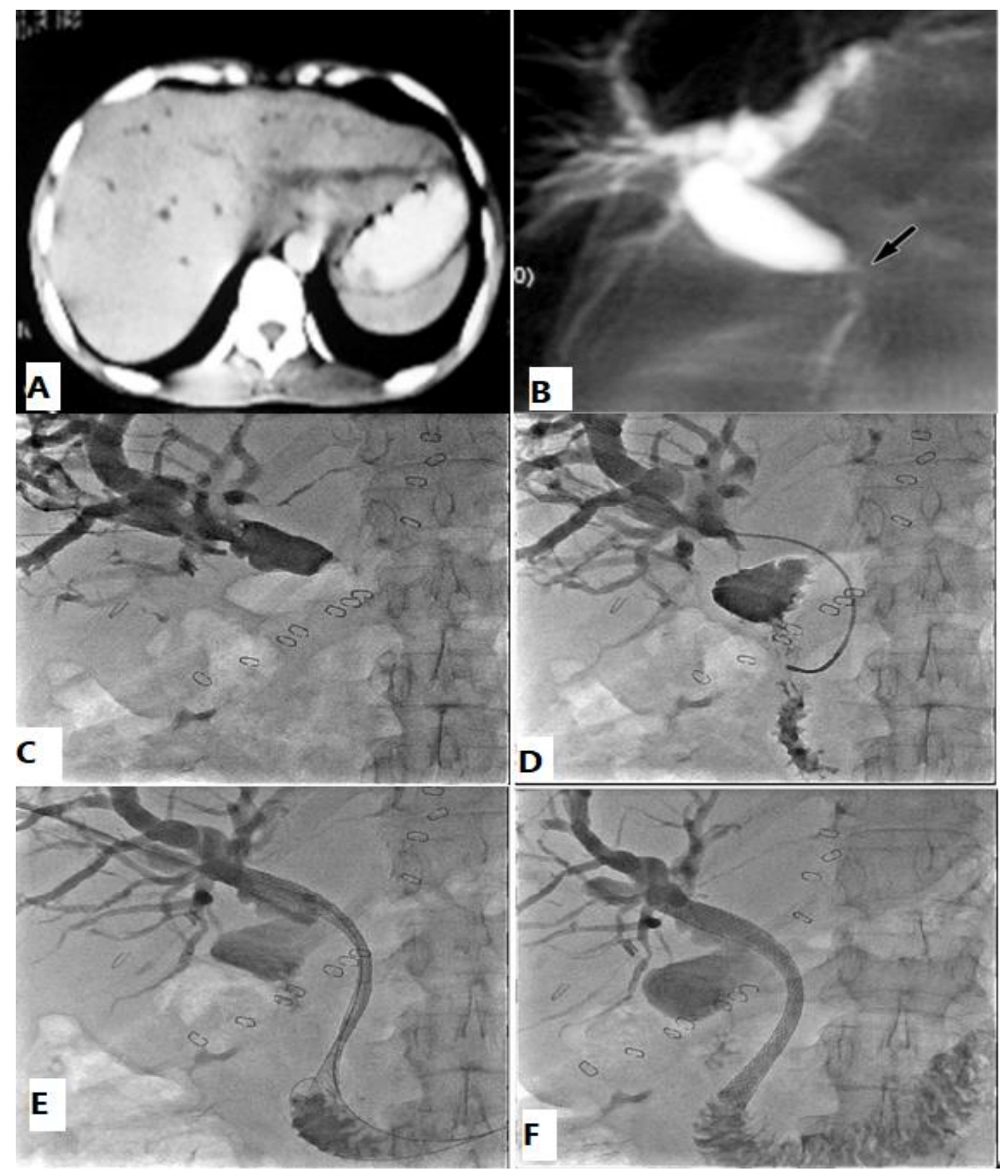

Figure (4): 49 years old female patient with obstructive jaundice due to post cholecystectomy benign stricture. (A) Enhanced axial CT scan shows intrahepatic bile duct dilatation. (B) MRCP shows moderate intrahepatic and extrahepatic biliary dilatation caused by short tight stricture (arrow) of common bile duct where cystic duct origin once began. Intact distal bile duct segment is seen. (C \&D) PTC shows obstruction at the middle part of the CBD (Bisthmus type I obstruction) and guide wire is tried to pass the stricture, with pigtail catheter over it. contrast injection ensure that the guide wire and the catheter are traversing the stricture to the duodenum. (E) PTC shows the metallic stent was successfully placed over the guide wire traversing the stricture to the duodenum (F) PTC shows that the guide wire was removed leaving the successfully placed stent and flow of contrast through the obstructed segment to the duodenum. 


\section{$\underline{\text { Discussion }}$}

Percutaneous transhepatic cholangiography is a diagnostic and interventional therapeutic procedure used in the diagnosis and management of obstructive jaundice caused by benign and malignant biliary obstruction (Chandrashekhara et. al, 2016).

In this study 30 patients diagnosed with obstructive jaundice by clinical and laboratory methods. All patients were examined by US, CT and in some cases MRCP, finally PTC and some interventional procedures were done either as a therapeutic or palliative treatment for obstructive jaundice and temporary relieve of obstruction. The purpose of this study was to evaluate the therapeutic and diagnostic value of PTC and biliary drainage in patients with obstructive jaundice.

This study included 30 patients 21 male and 9 females, the mean age of patients was 59.1 \pm 6.3 years with range, $44-70$ years and these matched with Shukla et. al. they concluded that obstructive jaundice is more common in male than female and obstructive jaundice is more evident in 5th and 6th decade of life because age is considered as a risk factor for development of malignant obstructive jaundice (Shukla et. al. 2018).

Shukla et. al. concluded that malignant obstructive jaundice is more common than benign obstructive jaundice and this matched with the current study as $96.66 \%$ of patients in this study had malignant obstructive jaundice and only $3.33 \%$ of patients had benign obstructive jaundice (Shukla et. al. 2018).

Diagnosis of biliary obstruction is done by detecting the dilatation of intra or extrahepatic biliary channels, the cause and the level of obstruction, in this study the accuracy of US and CT in diagnosis of biliary dilatation were $100 \%$ for both techniques, their accuracy for diagnosis of cause of obstruction were $56.6 \%$ and $80 \%$ respectively, and their accuracy for diagnosis of level of obstruction were $46.6 \%$ and $66.6 \%$ respectively. The accuracy of PTC in diagnosis of biliary dilatation, cause and level of obstruction was 100\%, these was matched with Fekaj et. al. they concluded that accuracy of US and CT in diagnosis of biliary dilatation were $98 \%$ and $94 \%$ respectively and their accuracy for diagnosis of cause and level of obstruction were $47 \%$ and $92 \%$ respectively, they also concluded that The accuracy of PTC in diagnosis of biliary dilatation, cause and level of obstruction was $90-100 \%$ (Fekaj et. al. 2017).

The Bismuth-Corlette classified the hilar tumors into 4 types. Type I lesions are distal to the confluence of hepatic duct. Type II lesions involve and extend to the hepatic duct confluence. Type III lesions involve the hepatic duct confluence and either the right proximal hepatic duct (type IIIA) or left proximal hepatic duct (type IIIB). Type IV 
lesions extend into the both proximal hepatic ducts and segmental bile ducts (Soares KC et al 2015).

In current study according to the level of obstruction (bisthmus-correlate classification) the patients were classified to $73.33 \%$ of patients had type I obstruction, $3.33 \%$ had type II obstruction, $3.33 \%$ had type III obstruction and $20 \%$ had distal obstruction this was not matched with Yi et. al. they reported that the $50 \%$ of obstructive jaundice patients had type VI obstruction that was not included in our study (Yi et al 2015).

For management of obstructive jaundice and with the availability of percutaneous, endoscopic, and surgical approaches, the selection of the best modality used for biliary drainage depends mainly on the surgical options suitable for the patient at the presentation time. For the patients who are not surgical suitable due to non-resectability of lesion or to the risk of comorbidities, the choice of percutaneous procedure versus endoscopic one depends largely on the site and extent of the obstructing lesion and the operator experience (Garcarek et. al., 2012) (Coelen et. al. 2018).

Retrograde endoscopic stenting is considered as the procedure of choice for stenting of biliary stricture, but in some patients, it is not possible to pass the malignant stricture by ERCP. For these patients percutaneous stenting is considered this was proved by Zhao et. al. they concluded that in non-operable biliary obstruction, endoscopic drainage is recommended but if it is unavailable or has failed, percutaneous drainage procedure is recommended. Also, PTBD is recommended for hilar biliary obstruction while ERCP is recommended in distal biliary obstruction (Zhao et.al. 2015).

Successful biliary drainage by PTC occured in 30 patients $(100 \%)$ included in this study with technical success rate for passing the stricture was $86.66 \%$ this was matched with Dawoud et. al. they proved that the technical success rate for passing the stricture was 81.2\% (Dawoud et. al. 2017).

In current study metallic stent was used in 21 patients (70\%) and plastic stent in 5 patients these was due to the advantages of metallic stent over plastic stent as metallic stent has wider diameter so decrease the possibility of occlusions so it decrease the need for re-intervention (Davids et. al. 2013) (Hannesson et. al. 2013).

In current study right hepatic lobe approach was done in $80 \%$ of patients, left hepatic lobe approach was done in $6.66 \%$ of patients and both approaches done in $13.33 \%$ of patients. Chandrashekhara et. al. reported that selection of right or left biliary approach depends on the hepatic lobe volume, presence of cholangitis and portal vein status. The right hepatic lobe is preferred in PTBD because it has the ability to save the functioning parenchyma of the liver (Chandrashekhara et. al, 2016). 
In this study there were significant decrease of total bilirubin, and alkaline phosphatase levels and improvement of liver function tests was seen in all 30 patients following 1 week after percutaneous biliary drainage with successful percutaneous biliary drainage occurred in 30 patients $(100 \%)$ by means of percutaneous external biliary drainage and/or biliary stenting. This was higher than the results of Dawoud et. al. they reported that therapeutic success rate of percutaneous biliary drainage was 70\% (Dawoud et.al. 2019).

In this study technique-related complications occurred in 4 patients of all 30 cases with technique related morbidity was $13.33 \%$ this was lower than the results of Dawoud et. al. they reported that complication occurred in $20 \%$ of the patients (Dawoud et.al. 2019).

The technique related complication in this study included bile leakage and subsequent bile peritonitis, but this controlled by adequate medical treatment Chandrashekhara et. al. reported that peri-catheter bile leak is a common complication. It is caused by side holes of catheter located outside the biliary system, ascites, catheter block or kinking (Chandrashekhara et. al. 2016).

Another technique related complication in current study was acute cholangitis that occurred seven days after the procedure and it controlled by medical treatment. Ahn et. al. reported that exact etiology of cholangitis is unknown, but it may be due to many factors like retrograde reflux of intestinal flora during the technique, external infection along the drainage catheter, or due to hematogenous infection (Ahn et. al. 2013).

Another technique related complications in this study were a self-limiting subcapsular liver hematoma and hemobilia due to injury of right hepatic artery during PTC needle insertion which was immediately embolized with metallic coils. Chandrashekhara et. al. reported that post PTBD hemobilia or hemmorage is less common and transient complication and is seen more with puncture of peripheral biliary radicle. Also branches of portal vein and hepatic artery accompany the dilated bile ducts, and the side holes of the catheter may be wrongly positioned in these vessels (Chandrashekhara et. al. 2016).

In this study occlusion of the stent occurred in 8/26 patients $(30.07 \%)$, occlusion was caused by tumor growth in six patients and due to sludge incrustation in two cases. Chandrashekhara et. al. reported that stent occlusion or blockage is considered as a delayed complication of PTBD. Occlusion of the stent may be caused by tumor regrowth (Chandrashekhara et. al. 2016). 


\section{Conclusion}

Percutaneous trans-hepatic cholangiography has high diagnostic accuracy rate 100\% in diagnosis of biliary dilatation, cause and level of biliary obstruction. Percutaneous transhepatic biliary drainage has high therapeutic success rate of bile drainage $100 \%$ with high technical success rate $86.66 \%$ for passing the stricture in patients with obstructive jaundice.

In our study technique-related morbidity was $13.33 \%$, with a procedure-related mortality rate of $0 \%$.

\section{$\underline{\text { Abbreviations }}$}

PTC: Percutaneous trans-hepatic cholangiography.

CBD: Common Bile Duct.

CT: Computed Tomography.

MRCP: Magnetic Resonance Cholangio-pancreatography.

ERCP: Endoscopic Retrograde Cholangio-Pancreatography.

US: Ultrasound.

PTBD: percutaneous biliary drainage

\section{$\underline{\text { References }}$}

- Ahmed O, Mathevosian S, and Arslan B (2016). Biliary Interventions: Tools and Techniques of the Trade, Access, Cholangiography, Biopsy, Cholangioscopy, Cholangioplasty, Stenting, Stone Extraction, and Brachytherapy. Semin Intervent Radiol ;33:283-290

- Ahn S, Lee Y-S, Lim KS, et al. (2013). Malignant biliary obstructions: can we predict immediate postprocedural cholangitis after percutaneous biliary drainage? Support Care Cancer;21:2321-6.

- Altman A and Zangan SM (2016). Benign Biliary Strictures. Semin Intervent Radiol; 33:297-306

- Barkay O, Mosler P, Schmitt CM, et al. (2013). Effect of endoscopic stenting of malignant bile duct obstruction on quality of life. J Clin Gastroenterol;47:526-31. 
- Chandrashekhara SH, Gamanagatti S, Singh A, and Bhatnagar S (2016). Current Status of Percutaneous Transhepatic Biliary Drainage in Palliation of Malignant Obstructive Jaundice: A Review. Indian Journal of Palliative Care; Vol 22 / Issue 4.

- Coelen RJS, Roos E, Wiggers JK, et al. (2018). Endoscopic versus percutaneous biliary drainage in patients with resectable perihilar cholangiocarcinoma: a multicentre, randomised controlled trial. Lancet Gastroenterol Hepatol;3:681-90.

- Crosara Teixeira M, Mak MP, Marques DF, Capareli F, et al (2013). Percutaneous transhepatic biliary drainage in patients with advanced solid malignancies: Prognostic factors and clinical outcomes. J Gastrointest Cancer; 44:398-403.

- Davids P.H.P., Groen A.K., Rauws E.A.J., et al. (2013). Randomised trial of selfexpanding metal stents versus polyethylene stents for distal malignat biliary obstruction. Lancet, 340: 1488-92.

- Dawoud AM,. Omar HM, Amin MA and Nooman A (2019). Radiological Intervention and Imaging Procedures in Management of Patients with Malignant Obstructive Jaundice. Med. J. Cairo Univ., Vol. 87, No. 5,: 2791-2800.

- Fekaj E, Jankulovski N and Matveeva N (2017). Obstructive Jaundice. Austin Dig Syst. 2(1): 1006.

- Garcarek J, Kurcz J, Guzinski M, Janczak D, Sasiadek M (2012). Ten years single center experience in percutaneous transhepatic decompression of biliary tree in patients with malignant obstructive jaundice. Adv Clin Exp Med; 21: 621-32.

- Hannesson P.H., Sandberg A., Stridbeck H., et al. (2013). Treatment of malignant biliary obstruction by percutaneous transhepatic insertion of expomdable metallic stents, 2013, European Radiology, 5: 1-5.

- Ray C.E., Lorenz J.M., Burke C.T., et al. (2013). ACR Appropriateness Criteria radiologic management of benign and malignant biliary obstruction. J. Am. Coll Radiol.,10: 567-74.

- Rees J, Mytton J, Evison F, Mangat KS et.al (2020). The outcomes of biliary drainage by percutaneous transhepatic cholangiography for the palliation of malignant biliary obstruction in England between 2001 and 2014: a retrospective cohort study. BMJ; 10:e033576.

- Shawyer A,Goodwin MD and Gibson RN (2013). Interventional biliary radiology: current state-of-the-art and future directions. Imaging Med; 5(6), 525538.

- Shukla S, Kharat PR, Patbamniya N, Kumar K (2018). Clinicopathological study on patients presenting with obstructive jaundice. International Surgery Journal. 5(2):705-710. 
- Soares KC, Kamel I,. Cosgrove DP, Herman JM et. al. (2014). Hilar cholangiocarcinoma: diagnosis, treatment options, and management. Hepatobiliary Surgery and Nutrition, 3(1): 18-34.

- Tuqan W, Innabi A, Alawneh A, Abu Farsakh A, et.al.(2017). Prediction of survival following percutaneous biliary drainage for malignant biliary obstruction. Journal of Translational Internal Medicine; Vol 5 | Issue 2.

- Yi S, Cui X, Xiong L, Deng X, Pei D et. al. (2015). Prognostic Value of Bismuth Typing and Modified T-stage in Hilar Cholangiocarcinoma. Cancer Translational medicine. Volume : 1 Issue : 1,1-5.

- Zhao X-qian, Dong J-hong, Jiang K, et al. (2015). Comparison of percutaneous transhepatic biliary drainage and endoscopic biliary drainage in the management of malignant biliary tract obstruction: a meta-analysis. Dig Endosc; 27:137-45. 